\title{
Assessment of Renal Ischemia By Optical Spectroscopy
}

J. T. Fitzgerald, S. Demos, A. Michalopoulou, J. L. Pierce, C. Troppmann

February 6, 2004

Journal of Surgical Research 
This document was prepared as an account of work sponsored by an agency of the United States Government. Neither the United States Government nor the University of California nor any of their employees, makes any warranty, express or implied, or assumes any legal liability or responsibility for the accuracy, completeness, or usefulness of any information, apparatus, product, or process disclosed, or represents that its use would not infringe privately owned rights. Reference herein to any specific commercial product, process, or service by trade name, trademark, manufacturer, or otherwise, does not necessarily constitute or imply its endorsement, recommendation, or favoring by the United States Government or the University of California. The views and opinions of authors expressed herein do not necessarily state or reflect those of the United States Government or the University of California, and shall not be used for advertising or product endorsement purposes. 


\section{Assessment of Renal Ischemia By Optical Spectroscopy}

Jason T. Fitzgerald, M.D. ${ }^{1}$

Stavros Demos, Ph.D. ${ }^{2}$

Andromachi Michalopoulou, Ph.D. ${ }^{3}$

Jonathan L. Pierce, M.D. ${ }^{1}$

Christoph Troppmann, M.D. ${ }^{1}$

${ }^{1}$ Department of Surgery, University of California, Davis Medical Center, Sacramento, CA, U.S.A.

${ }^{2}$ Lawrence-Livermore National Laboratory, Livermore, CA, U.S.A.

${ }^{3}$ Department of Urology, University of California, Davis Medical Center, Sacramento, CA, U.S.A.

Acknowledgement:

The authors wish to thank Neil H. Willits of the U.C. Davis Statistical Laboratory for his assistance. Jeremy Johnson and James Tabibian provided valuable technical assistance with the experiments.

This work has been supported in part by funding from the Center for Biophotonics and the Lawrence Livermore National Laboratory through Grant No. (UCDRD Proposal \# LS03-009). The Center for Biophotonics, an NSF Science and Technology Center, is managed by the University of California, Davis, under Cooperative Agreement No. PHY 0120999.

This work was performed in part at UC, Lawrence Livermore National Laboratory under the auspices of the U.S. Department of Energy under Contract W-7405-Eng-48. 


\begin{abstract}
Introduction: No reliable method currently exists for quantifying the degree of warm ischemia in kidney grafts prior to transplantation. We describe a method for evaluating pretransplant warm ischemia time using optical spectroscopic methods.

Methods: Lewis rat kidney vascular pedicles were clamped unilaterally in vivo for 0, 5, $10,20,30,60,90$ or 120 minutes; 8 animals were studied at each time point. Injured and contralateral control kidneys were then flushed with Euro-Collins solution, resected and placed on ice. $335 \mathrm{~nm}$ excitation autofluorescence as well as cross polarized light scattering images were taken of each injured and control kidney using filters of various wavelengths. The intensity ratio of the injured to normal kidneys was compared to ischemia time.
\end{abstract}

Results: Autofluorescence intensity ratios through a $450 \mathrm{~nm}$ filter and light scattering intensity ratios through an $800 \mathrm{~nm}$ filter both decreased significantly with increasing ischemia time ( $\mathrm{p}<0.0001$ for each method, one-way ANOVA). All adjacent and non-adjacent time points between 0 and 90 minutes were distinguishable using one of these two modalities by Fisher's PLSD.

Conclusions: Optical spectroscopic methods can accurately quantify warm ischemia time in kidneys that have been subsequently hypothermically preserved. Further studies are needed to correlate results with physiological damage and posttransplant performance. 


\section{INTRODUCTION}

Whether or not a cadaveric donor's organs are suitable for transplantation can be a difficult question faced by transplant surgeons. Especially if the donor has experienced significant hemodynamic instability, organs may have suffered an amount of ischemia that is difficult to quantify. Variable amounts of warm ischemia also accrue during procurement of organs from both brain-dead and non heart-beating donors. Transplanting organs with significant pretransplant warm ischemic damage may leave the recipient with unacceptably poor allograft function and adversely affect graft and patient survival. On the other hand, as transplant waiting lists continue to grow, the ability to maximize utilization of viable organs from marginal donors becomes increasingly important. How much warm ischemic damage has been sustained by a potentially transplantable organ? Tests of vascular resistance (1), enzymatic leakage from cells (2), electrolyte fluctuations, and other ischemic changes (3) have been devised to answer this question, but unfortunately these tests are hindered by hypothermic preservation and by clinical practicality (4). Currently, the only reliable way of assessing the ischemic damage that an organ has suffered is to transplant it and monitor its function.

Optical spectroscopy can measure changes in tissue by quantifying differences in a tissue's spectral properties - its ability to absorb, emit, and scatter light at various wavelengths. Measurements can be made quickly and no manipulation of an organ, such as biopsy or attachment of perfusion tubing, is required. Because no enzymatic assays are involved, optical spectroscopic measurements may not be hindered by hypothermic preservation methods. Previous investigators have utilized the autofluorescent properties 
of NADH to assess ischemia/reperfusion injury during the ex-vivo phase of experimental hepatic and renal transplantation (5). Others have correlated kidney graft surface infrared intensity immediately after reperfusion with early postoperative function (6). All of these methods involve analyses occurring during or after transplantation. In the practice of clinical organ transplantation, however, the optimal time window for taking measurements of graft viability is during hypothermic preservation. No methods are available to reliably quantify warm ischemia occurring before transplantation in hypothermically preserved organs.

In this study, our aim was to determine whether optical spectroscopic methods employed during hypothermic preservation, prior to implantation, are capable of assessing the amount of warm ischemia sustained by a kidney before hypothermic preservation. We used two optical spectroscopic modalities - autofluorescence, which can quantify hypoxia-related tissue fluorophores, such as NADH (7), (8), (9), (10), (11), and light scattering, which can detect cellular and molecular tissue changes (12), (13), (14), (15), (16). Rat kidney vascular pedicles were unilaterally clamped for various times, after which the injured and contralateral (internal control) kidneys were hypothermically preserved. Laser autofluorescence and cross-polarized light scattering images at several wavelengths were taken from each kidney and its control, and the ratio of the image intensity of the injured to normal kidney was calculated. We hypothesized that the intensity ratio would correspond to the duration of warm ischemic damage sustained by each kidney. 


\section{METHODS}

\section{Renal ischemia and hypothermic preservation}

All animal procedures were approved by the University of California, Davis Animal Use and Care Committee. Adult male Wistar rats weighing 300-500g were anesthetized using $10 \mathrm{mg} / \mathrm{kg}$ sodium pentobarbital $(50 \mathrm{mg} / \mathrm{ml})$ injected intraperitoneally. Laparotomy was performed through a midline abdominal incision. 200 units of heparin sulfate were injected systemically via the inferior vena cava (IVC) to prevent thrombosis during renal artery and vein clamping. The left kidney and its vascular pedicle were then dissected free of surrounding fascia and fatty tissue, and the left renal vascular pedicle was clamped for $0,5,10,20,30,60,90$ or 120 minutes. Eight rats were studied at each of these eight time points (total number of experimental animals: 64). In all cases, the right kidney was left undisturbed, as an internal control, until the conclusion of the ischemic period, at which time the aorta and inferior vena cava were clamped above and below the level of the renal arteries and veins, respectively. The left kidney was then unclamped and a venting incision was made in the juxtarenal IVC. $4 \mathrm{ml}$ of Euro-Collins electrolyte solution for kidney preservation (Baxter Healthcare Corp., Round Lake, IL) at $4^{\circ} \mathrm{C}$ were injected into the aorta just proximal to the renal arteries to flush the kidneys. Bilateral nephrectomies were then performed and the kidneys stored in Euro-Collins solution and ice. Animals were sacrificed after nephrectomy. 


\section{Optical spectroscopic techniques}

Hypothermically preserved ischemic and control kidneys from each animal were placed adjacent to each other on ice for optical spectroscopic analyses. For each pair of kidneys, two spectroscopic methods - laser autofluorescence (Figure 1) and crosspolarized light scattering imaging (Figure 2) - were employed. The laser autofluorescence technique utilized laser illumination to excite tissue on the surface of both kidneys with a wavelength of $335 \mathrm{~nm}$. This laser illumination wavelength was obtained by second harmonic conversion of the tunable output of an optical parametric oscillator (Opotek, Inc., Carlsbad, CA) pumped at $366 \mathrm{~nm}$ by the third harmonic of a Nd:YAG laser. Excitation with this wavelength is known to excite tissue to a depth of approximately 100 $\mu \mathrm{m}$ (17). Resulting autofluorescent images from each pair of kidneys were captured by a liquid nitrogen charge coupled device (CCD) array detector (512 x 512 pixels, Roper Scientific, Trenton, NJ). A camera lens was used to project the images to the CCD camera while a set of filters was placed in front of the camera in order to spectrally resolve the autofluorescent images. These consisted of long pass filters (designated wavelength and all greater wavelengths), and $20 \mathrm{~nm}$ bandwidth narrow band filters. The long pass filters used were $395 \mathrm{~nm}$ or $725 \mathrm{~nm}$, while the narrow band filters were 450 $\mathrm{nm}, 500 \mathrm{~nm}$ or $600 \mathrm{~nm}$. Images were analyzed using commercially available computer software (Roper Scientific) to assess the ratio of autofluorescence intensity of the ischemic to the control kidneys. The same imaging system was used to employ the light scattering technique. A white light source was used for illumination, delivered to the 
sample by a fiber optic bundle. Before reaching the sample, the illumination was passed first through a near infrared (NIR) filter and then through a linear polarizer. The net output provided polarized NIR illumination of the kidneys. Cross-polarized images were captured with a second polarizer located in front of the imaging system, oriented orthogonally with respect to the first polarizer located at the output of the illumination fiber. The remaining back scattered light was passed through $700 \mathrm{~nm}$ or $800 \mathrm{~nm}$ filters (both with a bandwidth of $10 \mathrm{~nm}$ ) to be captured by the CCD camera. The ratio of light scattering intensity of the ischemic to control kidneys was assessed using computer software (Roper Scientific). NIR light is known to penetrate tissue to a much greater depth than the laser described above (17), on the order of a few centimeters, with the light reaching greater depths losing its polarization by becoming scattered or absorbed. Structural changes at the cellular or even molecular level can cause changes in the scattering and absorption coefficients, leading to differences in back scattering intensity.

\section{Statistical analyses}

For both spectroscopic methods, the intensity ratio of the injured to the normal kidney was correlated with ischemia time. One-way ANOVA was used to assess the overall association of intensity ratios with ischemia time. Fisher's protected least significant difference (PLSD) method, adjusted for multiple comparisons, was used for pairwise comparisons of intensity ratios at different time points in order to determine the ability of the applied method to discern differences in individual points over the tested range of ischemia times. P-values of less than or equal to 0.05 were considered 
significant. Statistical analyses were performed using Statview for Windows (version 5.01, Abacus Concepts, Inc., Berkeley, CA).

\section{RESULTS}

The duration of hypothermic preservation before image acquisition ranged between 30 and 180 minutes. Figure 3 shows representative autofluorescence images of normal kidneys and of kidneys with 5 and 120 minutes of warm ischemia. The best overall differentiation in normal/injured intensity ratio with respect to ischemia time was achieved with autofluorescence images captured through the $450 \mathrm{~nm}$ filter (F-value 56.75, $\mathrm{p}<0.0001$, one-way ANOVA). Figure 4 displays the normal/injured intensity ratios plotted as a function of ischemia time using this filter. Although F-values obtained using the other filters were not as high, they were still highly significant: F-values for the $395 \mathrm{~nm}$ long pass, $500 \mathrm{~nm}$ narrow band, $600 \mathrm{~nm}$ narrow band and $725 \mathrm{~nm}$ long pass filters were $17.77(\mathrm{p}<0.0001), 17.28(\mathrm{p}<0.0001), 21.85(\mathrm{p}<0.0001)$, and $23.99(\mathrm{p}<$ 0.0001), respectively. Table 1 shows the results of Fisher's PLSD pairwise comparisons between normal/injured intensity ratios between all time points for autofluorescence images using the $450 \mathrm{~nm}$ filter. Pairwise comparisons were significantly different between adjacent ischemia time points of 0 and 5 minutes, 10 and 20 minutes, and 30 and 60 minutes The difference between 5 and 10 minutes approached statistical significance $(\mathrm{p}=0.0728)$. As shown in Table 1, the differences between most non-adjacent time points (5 and 20 minutes, for example) were significant. 
Figure 5 shows representative cross-polarized light scattering images of normal kidneys and of kidneys with 5 and 120 minutes of warm ischemia. Using this technique, the best differentiation was achieved with images captured through the $800 \mathrm{~nm}$ filter (Fvalue 17.72, $\mathrm{p}<0.0001$, one-way ANOVA). Figure 6 displays the normal/injured intensity ratios plotted as a function of ischemia time using this filter. The F-value using the $700 \mathrm{~nm}$ filter was $4.28(\mathrm{p}=0.002)$. Table 2 shows the results of Fisher's PLSD pairwise comparisons between normal/injured intensity ratios between all time points for cross-polarized light scattering images using the $800 \mathrm{~nm}$ filter. Pairwise comparisons were significantly different between the adjacent ischemia time points of 20 and 30 minutes and 60 and 90 minutes. As shown in Table 2, the differences between most nonadjacent time points (10 and 30 minutes, for example) were significant.

All adjacent and non-adjacent time points between 0 and 90 minutes were distinguishable by either autofluorescence imaging at $450 \mathrm{~nm}$ or light scattering imaging at $800 \mathrm{~nm}$.

\section{DISCUSSION}

As transplant donor criteria are expanded and organ allocation schemes become more complex, a method for detecting the degree of warm ischemic organ damage becomes increasingly relevant. The development of such a protocol could lead to more 
efficient use of organs by helping to select successfully transplantable organs from marginal donors and by avoiding transplantation of excessively damaged organs. Delayed graft function might also be predicted and dealt with preemptively. Currently available methods of assessing warm ischemic damage are hindered by hypothermic preservation methods (enzymatic assays and measurements of true vascular resistance), trauma to the allograft (e.g. biopsies), and clinical practicality (time consuming laboratory analyses).

We studied a noninvasive method for the quantification of warm ischemia in rat kidneys utilizing both autofluorescence and light scattering techniques. In the current clinical practice of organ transplantation, the amount of warm ischemic damage that has occurred in a potentially transplantable organ is the guess of the surgeon. The most practical time window in which to assess an organ's viability is after procurement and during hypothermic preservation, before transplantation into the recipient. During this time the organ is relatively protected from further ischemic damage and there may be a delay while the recipient is readied for surgery. To more closely approximate this clinical scenario, we followed the warm ischemia in our rat model by hypothermic preservation and subsequently by optical spectroscopic analysis. For both autofluorescence and light scattering techniques, the image intensity ratio of ischemically injured to normal kidneys decreased with increasing warm ischemia time; these changes were consistent and reproducible. We observed the greatest differences in intensity ratios in relation to warm ischemia time with autofluorescence images taken through a $450 \mathrm{~nm}$ filter and light scattering images taken through an $800 \mathrm{~nm}$ filter. Pairwise comparisons between time points indicated that both modalities combined were unable to detect differences between 90 and 120 minutes of ischemia time. A rational approach to 
quantifying the degree of unknown warm ischemia time would be to assess intensity ratios between the injured kidney and the control kidney using both the $450 \mathrm{~nm}$ autofluorescence and the $800 \mathrm{~nm}$ light scattering methods and plotting the obtained values into standardized curves.

The advantage of using autofluorescence and light scattering images, rather than a single point spectroscopic measurement, is that the intensity from the entire kidney could be analyzed as a single measurement. Thus, meaningful results can be obtained despite heterogeneity in tissue structure and in the degree of ischemia from point to point on the surface of the kidney. In contrast, spectral point measurements can analyze only a very small area of the kidney at one time. Because of the heterogeneity of injury across the kidney, data taken from one small point may not represent the whole surface; as a result, multiple measurements must be taken and averaged.

The short cold ischemia times, which varied from 30 to 180 minutes for each kidney pair in this experiment, are unlikely to have had a significant impact on the measurements of warm ischemia, since the optical spectroscopic measurements employed an intensity ratio of the injured to normal kidney images. During these experiments, no changes in intensity ratios due to cold ischemia were observed. Moreover, very little ischemic damage occurs during the first few hours of hypothermic kidney preservation in rat and human kidneys (18), (19), (20).

Optical spectroscopy for tissue analysis was introduced by Chance and Williams in 1955 and has involved measuring changes in tissue fluorophores such as mitochondrial $\mathrm{NADH}$, an adenine species normally oxidized during the course of cell respiration and ATP production (7), (8), (9), (10), (11). In contrast to autofluorescence analysis, in which 
specific substances (such as NADH) can be quantified based on their fluorescent properties, light scattering techniques are useful for measuring cellular and molecular structural changes in tissue due to ischemia: these changes affect the amount of light back scattered and recorded by the imaging system (12), (13). Currently, the most widely used clinical application of light scattering optical spectroscopy is pulse oximetry, in which the molecular structural differences between oxyhemoglobin and deoxyhemoglobin cause differences in the amount of back scattered light at different wavelengths. These differences can be quantified to yield an accurate measurement of the degree of arterial hemoglobin saturation with oxygen (14), (15), (16).

However, optical spectroscopy has not previously been used to assess warm ischemia in hypothermically preserved kidneys. Furthermore, to our knowledge, this is the first study combining autofluorescence and light scattering techniques to obtain information about tissue ischemia.

$\mathrm{NADH}$ increases during hypoxia and is the predominant fluorescing fluorophore at $450 \mathrm{~nm}$ at physiological temperatures when excited with a wavelength of around 335 $\mathrm{nm}(21),(22)$. In this study, however, decreased autofluorescence with increased ischemia time was observed. It is possible that we observed the behavior of an autofluorescing species other than NADH. Flavoprotein, which increases with increasing tissue hypoxia (23), (24), is known to fluoresce at slightly higher wavelengths than NADH (25), (26). Furthermore, while the $450 \mathrm{~nm}$ filter gave the best results statistically, images taken through all of the tested filters $(450 \mathrm{~nm}, 500 \mathrm{~nm}$ and $600 \mathrm{~nm}$ narrow band and 395 and $725 \mathrm{~nm}$ long pass) gave very similar injured/normal ratios with respect to ischemia time. This result suggests that all of the tissue fluorophores in the range of 
wavelengths tested were behaving similarly in response to ischemia, possibly as a consequence of severe alterations in $\mathrm{pH}$, which are known to change the emission efficiency of fluorophores (27), (28).

In the $800 \mathrm{~nm}$ range, the light scattering techniques used in this study may have been influenced by residual blood inside the kidney. Despite the fact that the kidneys were flushed with cold preservation solution, it is impossible to remove all the residual blood from the kidneys in this model. However, changes in oxy- and deoxyhemoglobin would likely not continue to evolve over a 60-90 minute period. We therefore speculate that the light scattering techniques detect additional structural changes brought about by ischemia, such as cellular edema or lysis.

The clinical aim of any measurement of pretransplant warm ischemia is to help in predicting a kidney graft's viability and function after transplantation. For the further development of optical spectroscopy as a pretransplant predictor of organ damage and posttransplant function, two important steps remain. First, while the measurements taken in this study correlated with the degree of warm ischemia time, it would be important clinically to correlate with actual pathophysiological measurements of tissue damage, such as capillary leak or creatinine clearance. Given the degree of variability in biological systems and the different factors that influence how tissue will respond to ischemia for a specific duration of time, optical spectroscopic measurements may correlate even more closely with physiological ischemic damage than with ischemia time. Second, and most importantly, such measurements must be correlated with posttransplant renal function. Ideally, this would lead to an absolute threshold measurement distinguishing a viable from a non-viable kidney. 


\section{CONCLUSION}

Optical spectroscopy is capable of measuring the duration of warm ischemic damage in hypothermically preserved rat kidneys. The best results were obtained by using autofluorescence images at $450 \mathrm{~nm}$ and light scattering images at $800 \mathrm{~nm}$. Further studies are needed to correlate pretransplant optical spectroscopic measurements with actual physiological injury and with kidney function after transplantation. 


\section{REFERENCES}

1. Inman S, Osgood R, Plott W, Richardson B, Lewis R. Identification of kidneys subjected to pre-retrieval warm ischemic injury during hypothermic perfusion preservation. Transplant Proc 1998; 30 (5): 2309.

2. Kievit JK, Nederstigt AP, Oomen AP, Janssen MA, Schoot L, Kootstra G. Release of alpha-glutathione S-transferase (alpha GST) and pi-glutathione Stransferase (pi GST) from ischemic damaged kidneys into the machine perfusate-relevance to viability assessment. Transplant Proc 1997; 29 (8): 3591.

3. Kootstra G, Kievit JK, Heineman E. The non heart-beating donor. Br Med Bull 1997; 53 (4): 844.

4. Stubenitsky BM, Booster MH, Nederstigt AP, Kievit JK, Jacobs RW, Kootstra

G. Kidney preservation in the next millenium. Transpl Int 1999; 12 (2): 83.

5. Thorniley MS, Lane N, Simpkin S, Fuller B, Jenabzadeh MZ, Green CJ.

Monitoring of mitochondrial NADH levels by surface fluorimetry as an indication of ischaemia during hepatic and renal transplantation. Adv Exp Med Biol 1996; 388: 431.

6. Gorbach A, Simonton D, Hale DA, Swanson SJ, Kirk AD. Objective, real-time, intraoperative assessment of renal perfusion using infrared imaging. Am J Transplant 2003; 3 (8): 988.

7. Chance B, Williams GR. Respiratory enzymes in oxidative phosphorylation. I. Kinetics of oxygen utilization. J Biol Chem 1955; 217 (1): 383. 
8. Barlow $\mathrm{CH}$, Harken $\mathrm{AH}$, Chance B. Evaluation of cardiac ischemia by NADH fluroescence photography. Ann Surg 1977; 186 (6): 737.

9. Shehada R, Marmarelis, V., Mansour, H., Grundfest, W. Laser induced fluorescence attenuation spectroscopy: detection of hypoxia. IEEE Transactions on Biomedical Engineering 2000; 47 (3): 301.

10. Barlow C, Rorvik, D., Kelly, J. Imaging epicardial oxygen. Annals of Biomedical Engineering 1998; 26: 76.

11. Mayevsky A, Nakache R, Merhav H, Luger-Hamer M, Sonn J. Real time monitoring of intraoperative allograft vitality. Transplant Proc 2000; 32 (4): 684. 12. Ba AM, Guiou M, Pouratian N, et al. Multiwavelength optical intrinsic signal imaging of cortical spreading depression. J Neurophysiol 2002; 88 (5): 2726.

13. Tao L, Masri D, Hrabetova S, Nicholson C. Light scattering in rat neocortical slices differs during spreading depression and ischemia. Brain Res 2002; 952 (2): 290.

14. Mendelson Y, Cheung PW, Neuman MR, Fleming DG, Cahn SD. Spectrophotometric investigation of pulsatile blood flow for transcutaneous reflectance oximetry. Adv Exp Med Biol 1983; 159: 93.

15. Anderson NM, Sekelj P. Light-absorbing and scattering properties of nonhaemolysed blood. Phys Med Biol 1967; 12 (2): 173.

16. Schmitt JM, Mihm FG, Meindl JD. New methods for whole blood oximetry. Ann Biomed Eng 1986; 14 (1): 35. 
17. Tuchin V. Tissue Optics: Light scattering methods and instruments for medical diagnosis. Bellingham, WA: The International Society for Optical Engineering, 2000.

18. Dragun D, Hoff U, Park JK, et al. Prolonged cold preservation augments vascular injury independent of renal transplant immunogenicity and function. Kidney Int 2001; 60 (3): 1173.

19. Roodnat JI, Mulder PG, Van Riemsdijk IC, JN IJ, van Gelder T, Weimar W. Ischemia times and donor serum creatinine in relation to renal graft failure. Transplantation 2003; 75 (6): 799.

20. Smits JM, van Houwelingen HC, De Meester J, et al. Permanent detrimental effect of nonimmunological factors on long-term renal graft survival: a parsimonious model of time-dependency. Transplantation 2000; 70 (2): 317. 21. Ji S, Chance B, Nishiki K, Smith T, Rich T. Micro-light guides: a new method for measuring tissue fluorescence and reflectance. Am J Physiol 1979; 236 (3): C144.

22. Thurman RG, Lemasters JJ. New micro-optical methods to study metabolism in periportal and pericentral regions of the liver lobule. Drug Metab Rev 1988; 19 (3-4): 263.

23. Paddle BM. A scanning fluorometer for imaging ischaemic areas in traumatized muscle. J Trauma 1988; 28 (1 Suppl): S189.

24. Tanaka A, Kitai T, Iwata S, et al. Delayed oxidation of intramitochondrial pyridine nucleotide oxidoreduction state as compared with tissue oxygenation in human liver transplantation. Biochim Biophys Acta 1993; 1182 (3): 250. 
25. Huang S, Heikal AA, Webb WW. Two-photon fluorescence spectroscopy and microscopy of NAD(P)H and flavoprotein. Biophys J 2002; 82 (5): 2811.

26. Mayeno AN, Hamann KJ, Gleich GJ. Granule-associated flavin adenine dinucleotide (FAD) is responsible for eosinophil autofluorescence. J Leukoc Biol 1992; 51 (2): 172.

27. Gryczynski Z, Gryczynski I, Lakowicz JR. Fluorescence-sensing methods. Methods Enzymol 2003; 360: 44.

28. Lin HJ, Herman P, Lakowicz JR. Fluorescence lifetime-resolved $\mathrm{pH}$ imaging of living cells. Cytometry 2003; 52A (2): 77. 


\section{Figure Legends}

Figure 1: Schematic of the autofluorescence method used for measurement of ischemia. A laser source was used to excite tissue at the surface of a hypothermically preserved kidney. Resultant autofluorescence was passed through narrow bandwidth filters of 450 $\mathrm{nm}, 500 \mathrm{~nm}$, or $600 \mathrm{~nm}$, and long pass filters of 395 or $725 \mathrm{~nm}$.

Figure 2: Schematic of the light scattering method used for the measurement of ischemia. A polarized light source was used to illuminate a hypothermically preserved kidney with near infrared (NIR) light. Resultant back scattered light was passed first through a cross polarizer to eliminate remaining the polarized light. The remaining back scattered light was passed through a $700 \mathrm{~nm}$ or $800 \mathrm{~nm}$ filter and captured as an image.

Figure 3: Representative autofluorescence images. In each set of images, the kidney on the left is the uninjured internal control kidney. The kidney on the right was injured for 5 minutes (A) and 120 minutes (B).

Figure 4: Normal/injured intensity ratios plotted as a function of time for the laser autofluorescence modality using a $450 \mathrm{~nm}$ filter, with a line drawn between the mean intensity ratios at each time point. Standard deviation bars are displayed around the mean intensity ratio at each time point. 
Figure 5: Representative light scattering images. In each set of images, the kidney on the left is the uninjured internal control kidney. The kidney on the right was injured for 5 minutes (A) and 120 minutes (B).

Figure 6: Normal/injured intensity ratios plotted as a function of time for the light scattering modality using an $800 \mathrm{~nm}$ filter, with a line drawn between the mean intensity ratios at each time point. Standard deviation bars are displayed around the mean intensity ratio at each time point. 
Figure 1

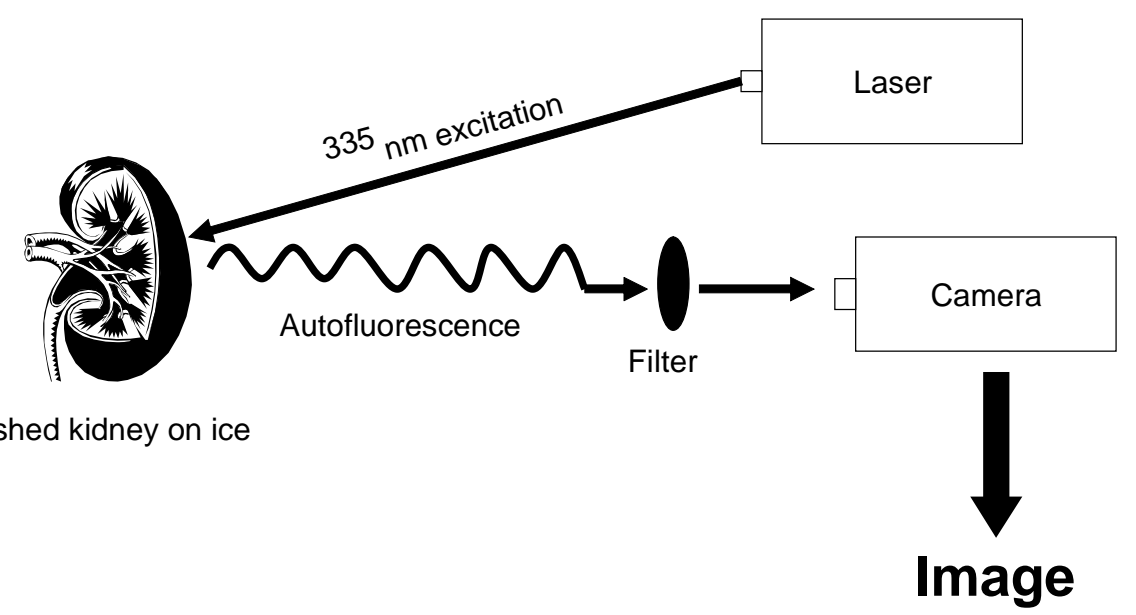

Figure 1

Figure 1

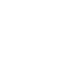

$$
\text { . }
$$

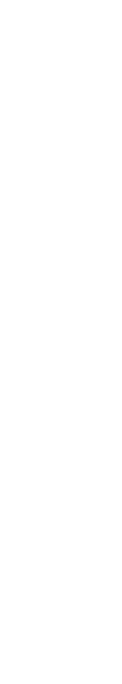

.

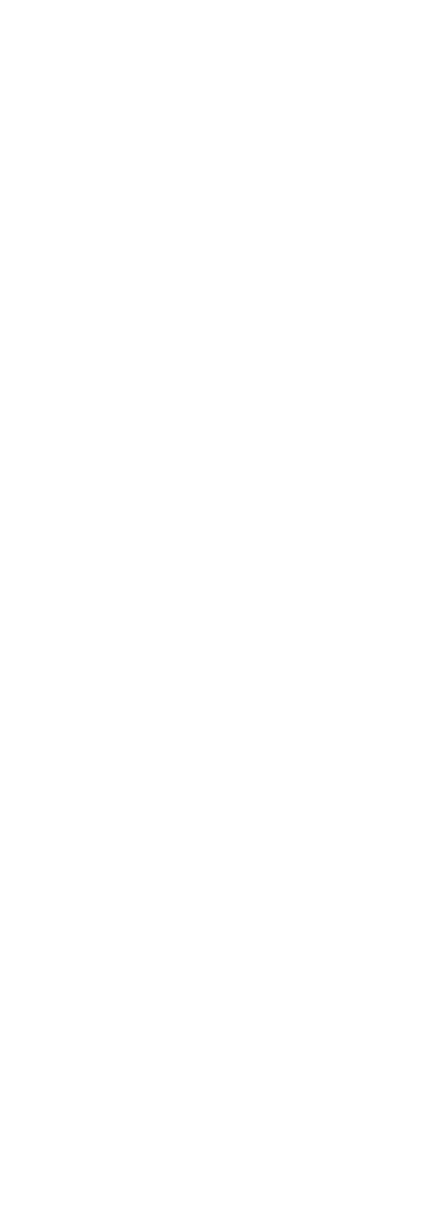

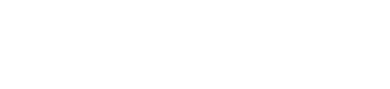


Figure 2

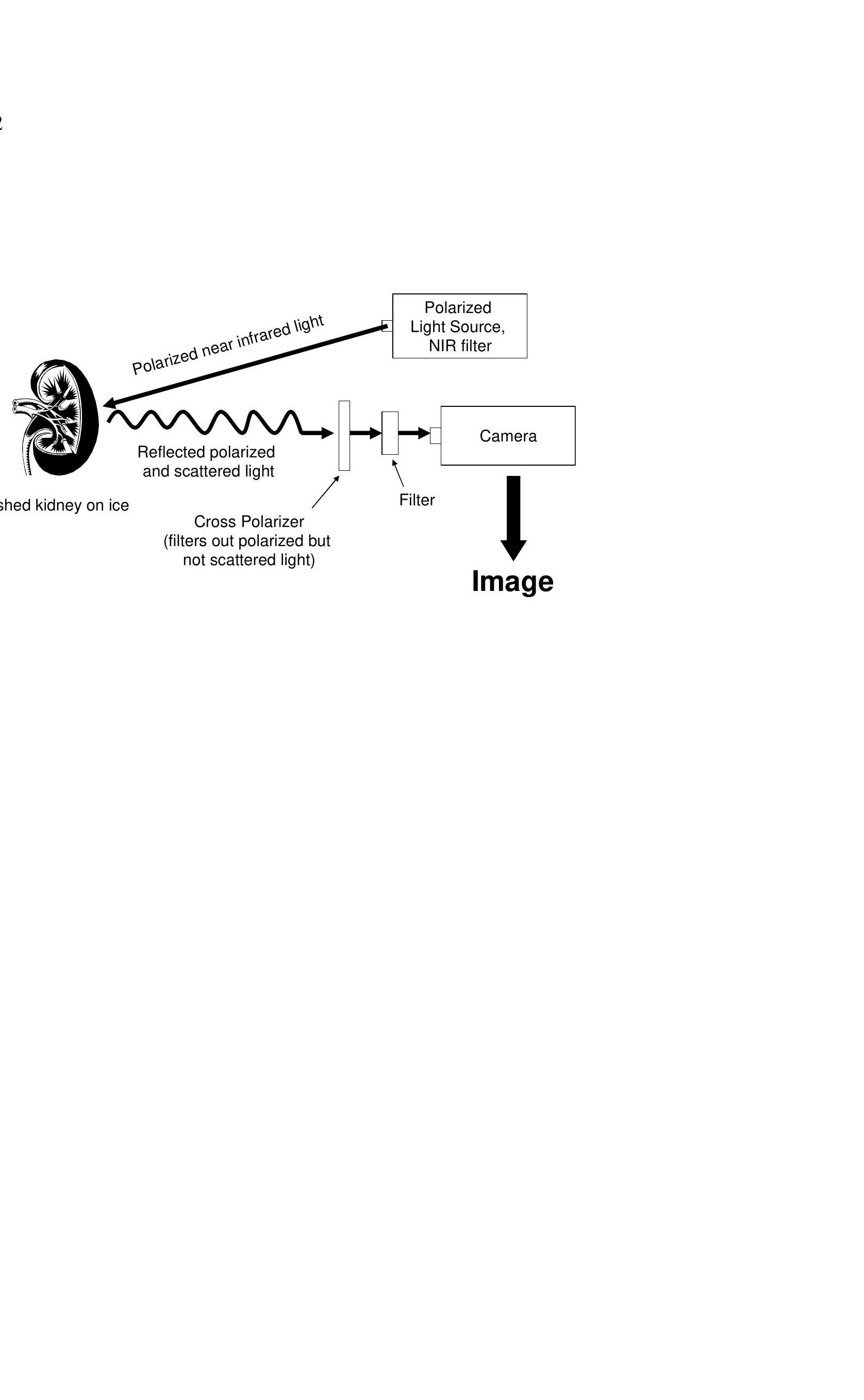

Figure 2

Figure 2

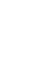

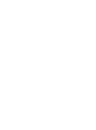

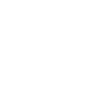

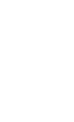

(2.

(1)

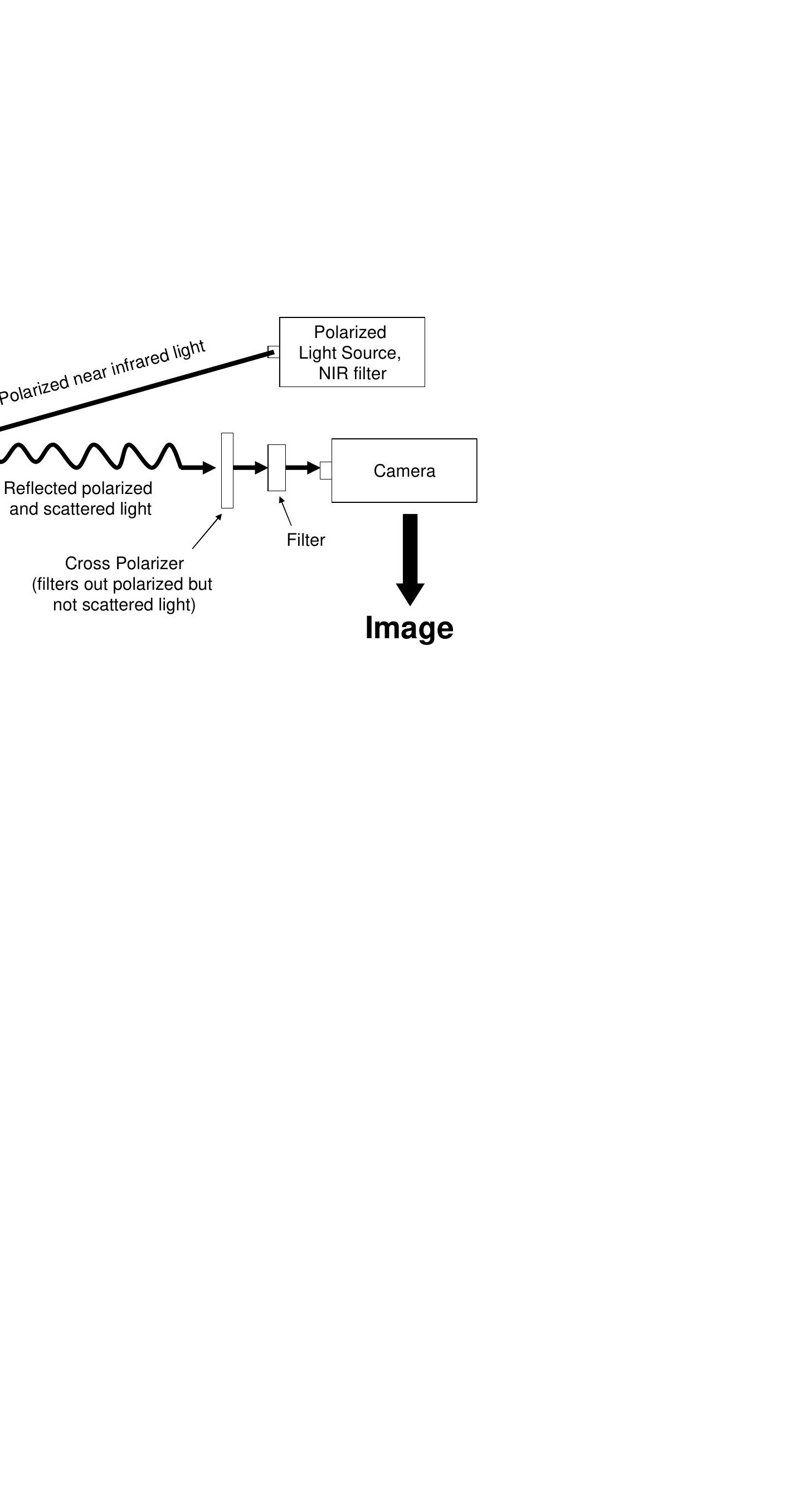

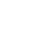

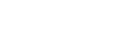
.
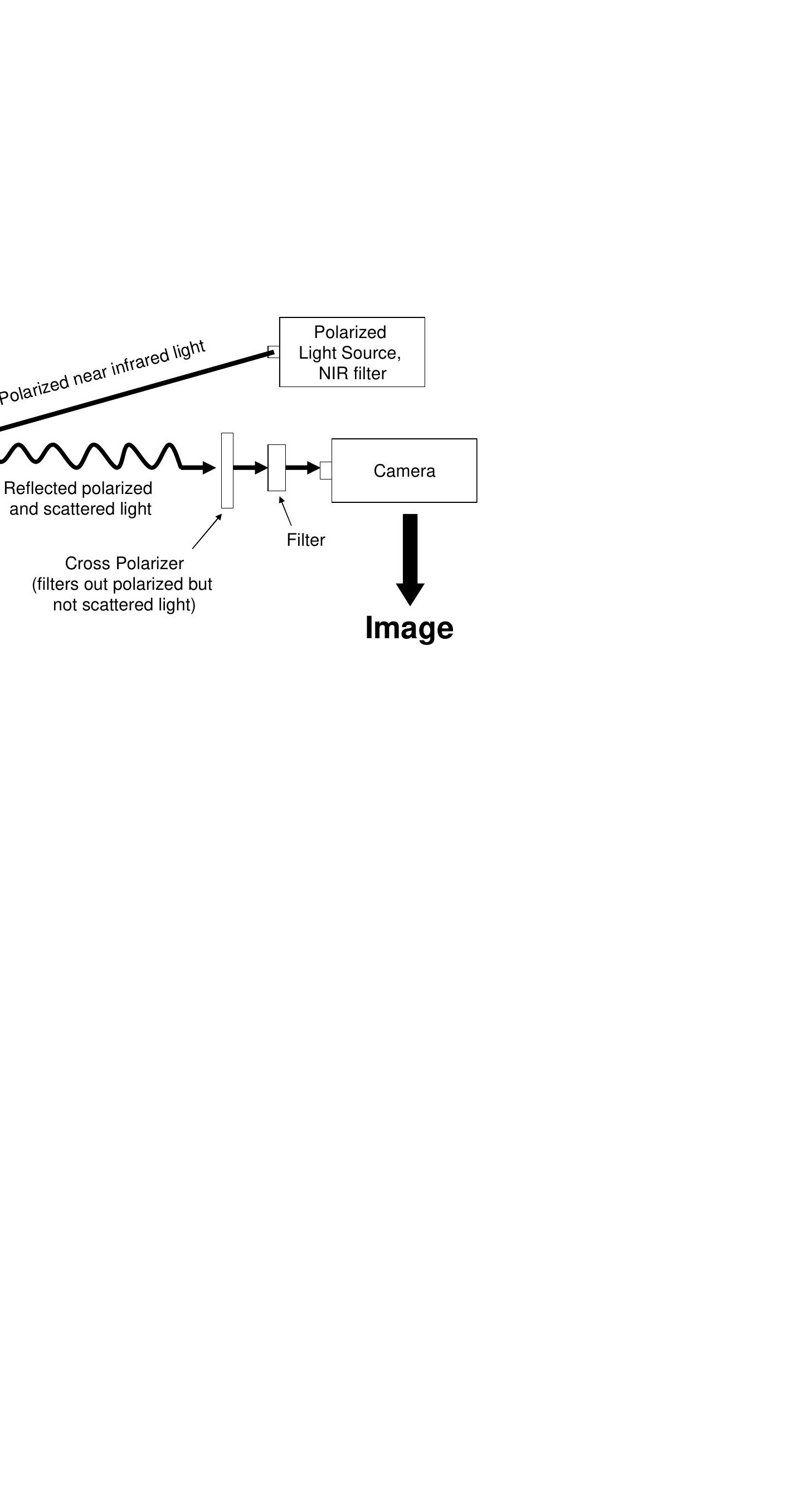
Figure 3
\[ \text { Figure 3: } \]

B.
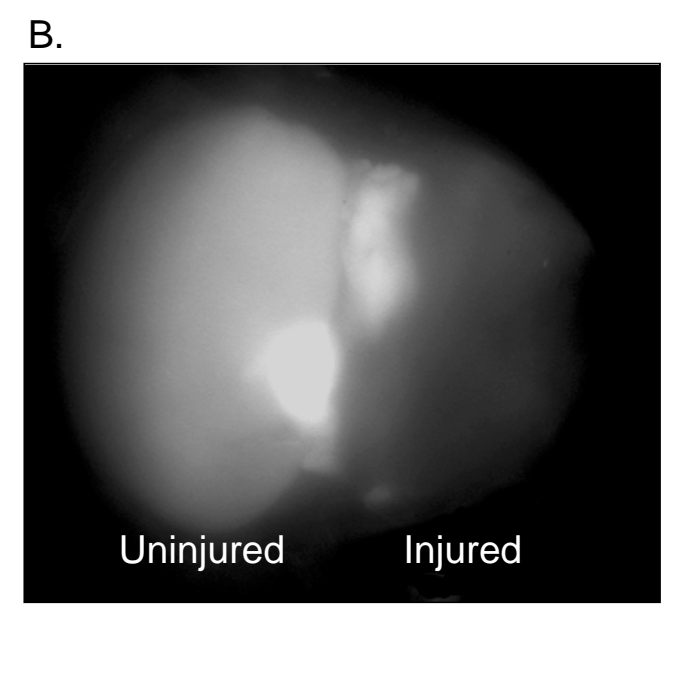

B.

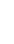

A.

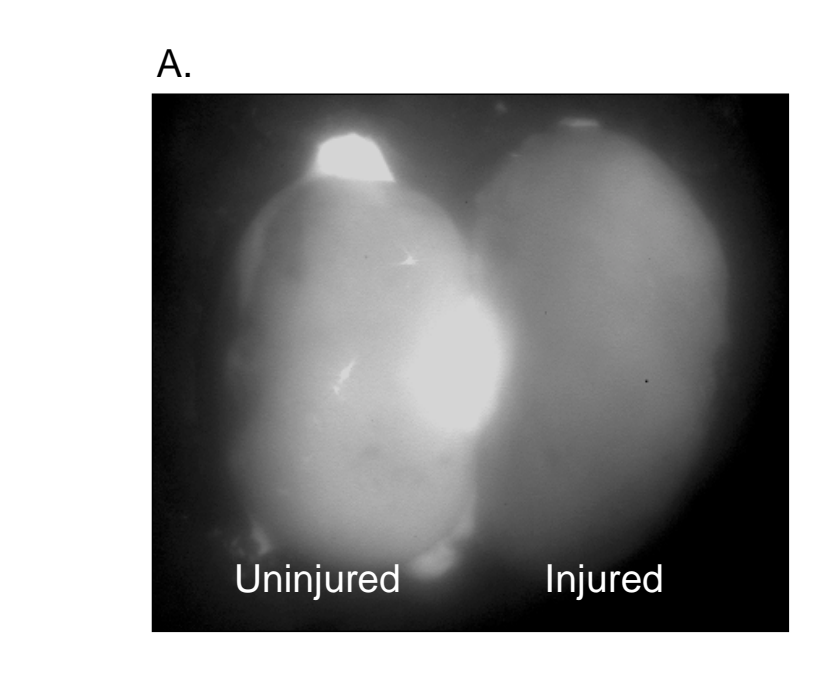


Figure 4:

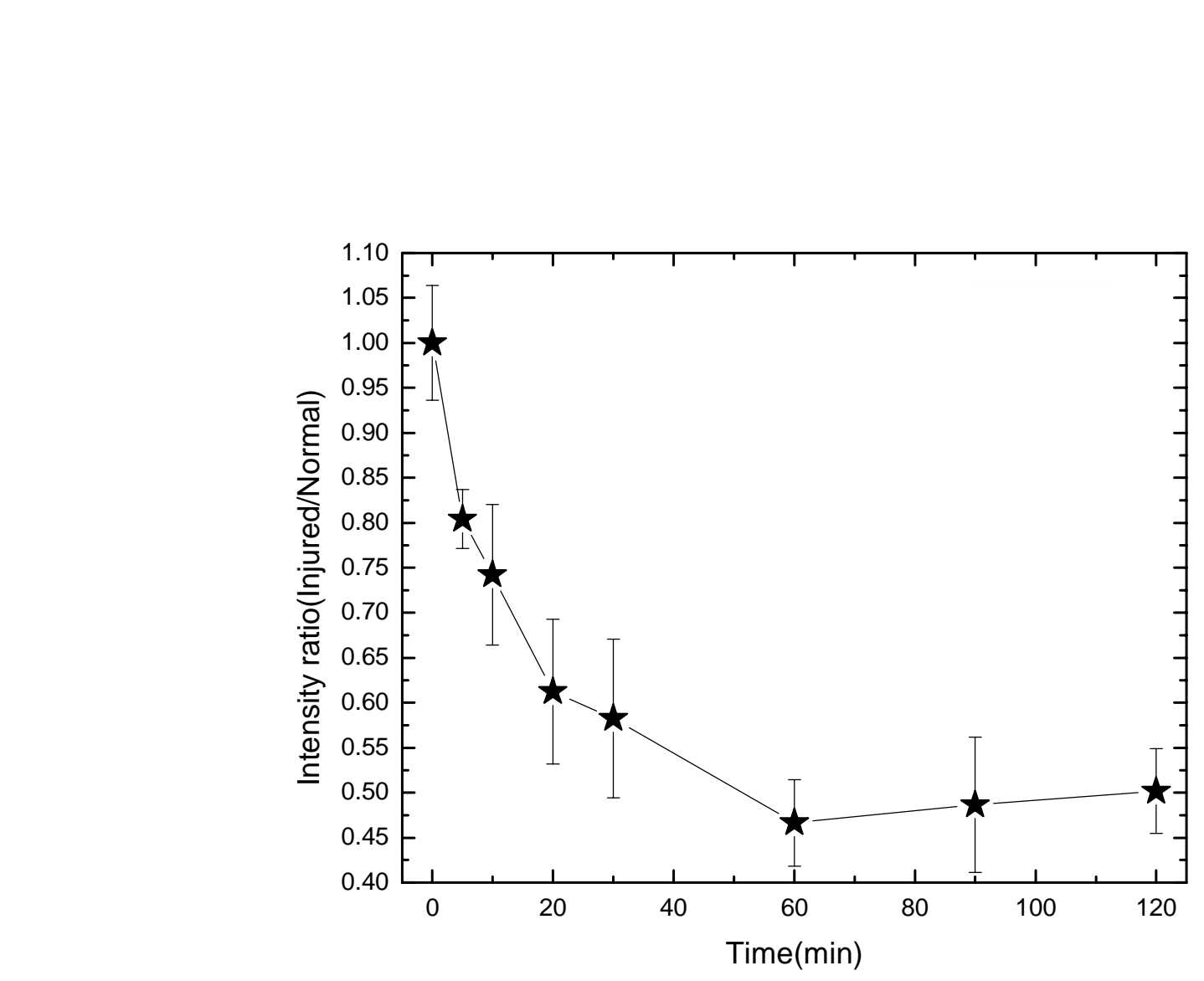

Figure 4

Figure 4:

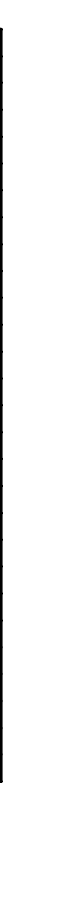

:

\section{Figure 4}

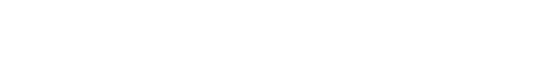

(1)
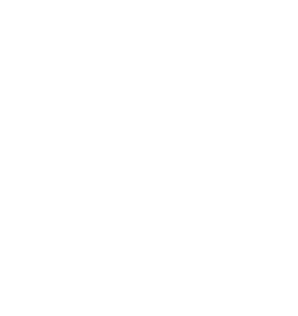


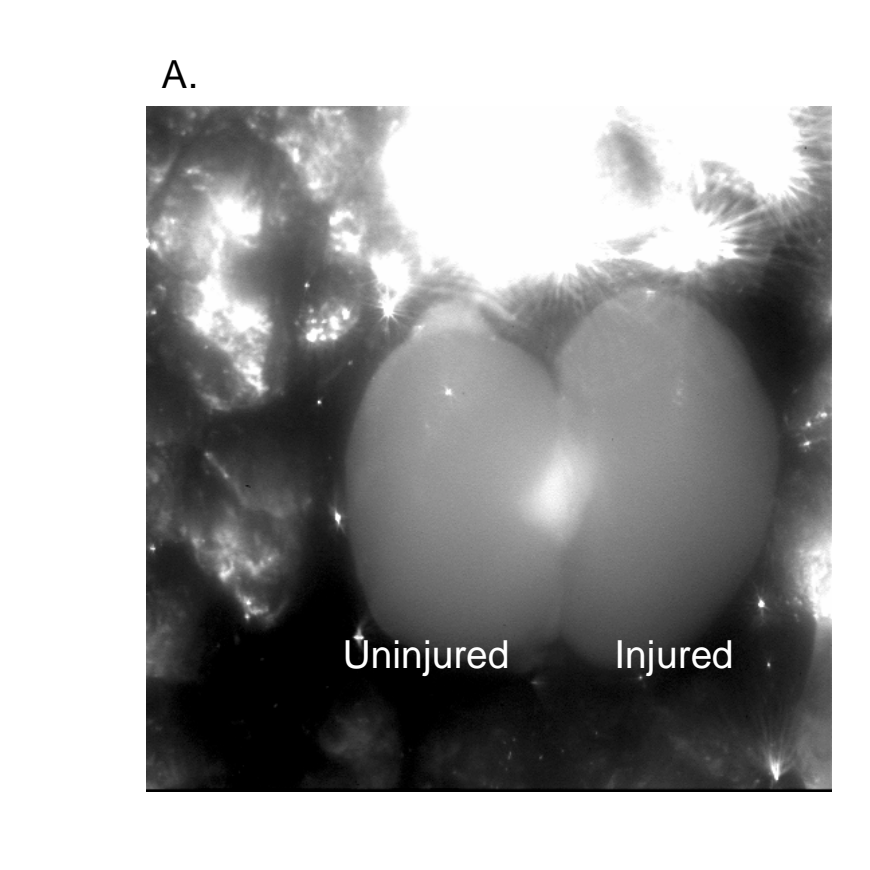

B.
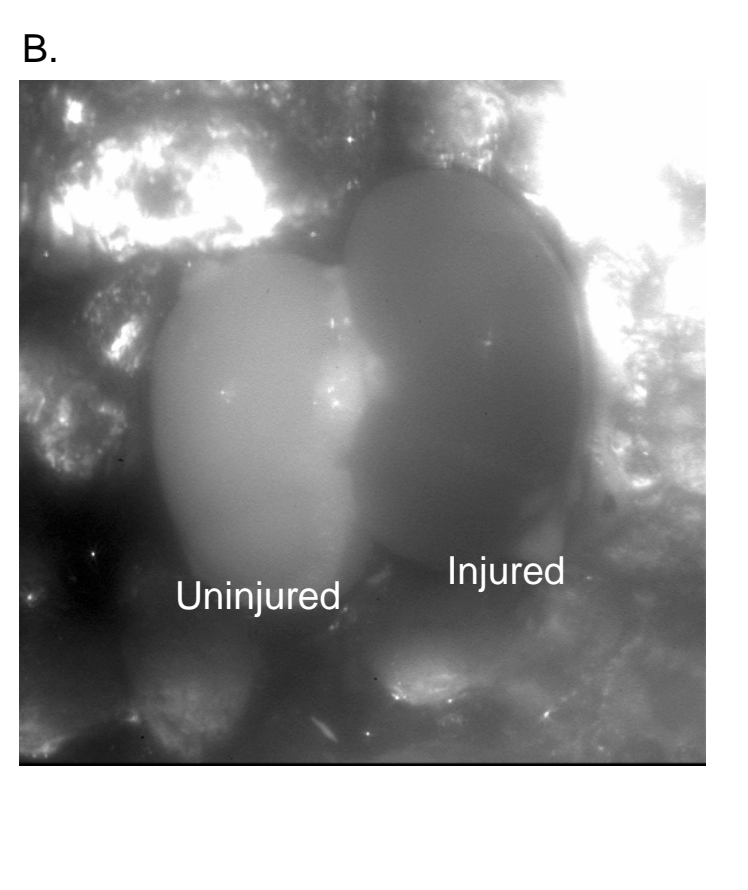

Figure 5
\[ \text { Figure 5: } \]

Figure 5
\[ \text { Figure } 5 \]

Figure 5
\[ \text { Figure 5: } \]

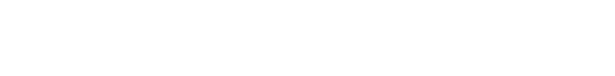

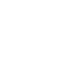

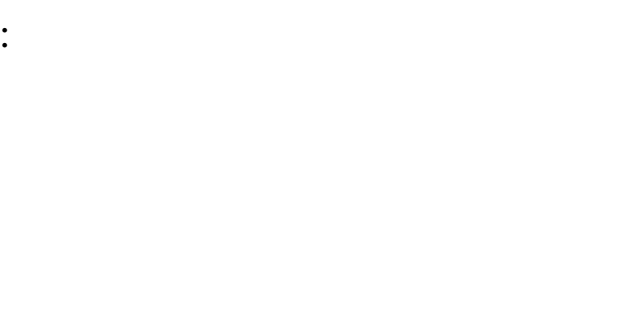


Figure 6:

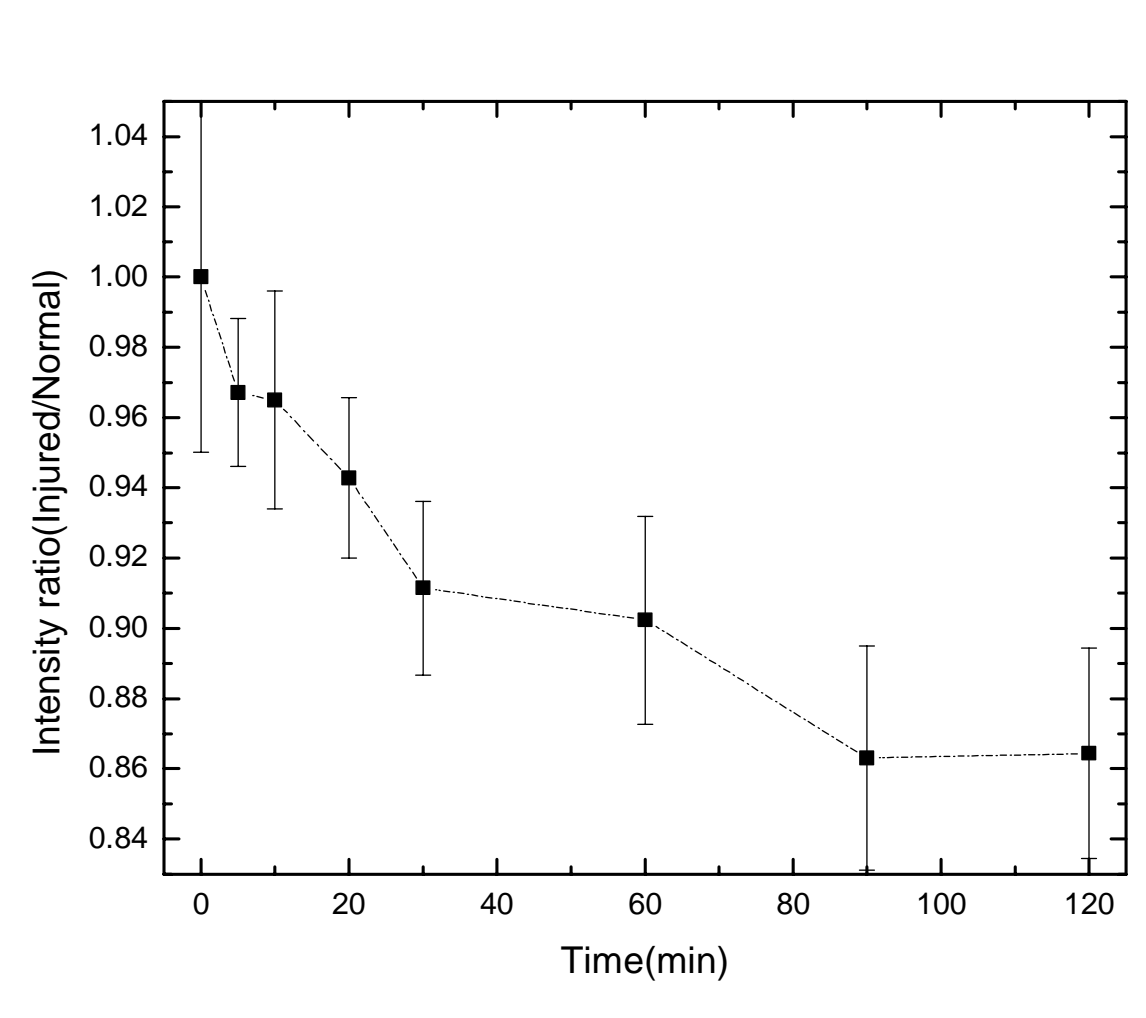

Figure 6

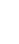

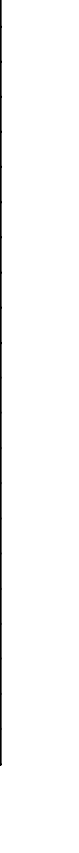


Table 1: Pairwise comparisons between time points for $450 \mathrm{~nm}$ autofluorescence intensity ratios. Shown are the mean differences in intensity ratios between time points, the critical differences required to achieve a p-value less than 0.05 , and the p-values as calculated by using Fisher's PLSD.

\begin{tabular}{|c|c|c|c|}
\hline Time Points (min.) & Mean Diff. & Critical Diff. & P-Value \\
\hline 0,5 & 0.209 & 0.070 & $<0.0001$ \\
\hline 0,10 & 0.271 & 0.070 & $<0.0001$ \\
\hline 0,20 & 0.401 & 0.072 & $<0.0001$ \\
\hline 0,30 & 0.431 & 0.070 & $<0.0001$ \\
\hline 0,60 & 0.547 & 0.072 & $<0.0001$ \\
\hline 0,90 & 0.527 & 0.075 & $<0.0001$ \\
\hline 0,120 & 0.512 & 0.072 & $<0.0001$ \\
\hline 5,10 & 0.062 & 0.067 & 0.0703 \\
\hline 5,20 & 0.192 & 0.070 & $<0.0001$ \\
\hline 5,30 & 0.222 & 0.067 & $<0.0001$ \\
\hline 5,60 & 0.338 & 0.070 & $<0.0001$ \\
\hline 5,90 & 0.318 & 0.073 & $<0.0001$ \\
\hline 5,120 & 0.302 & 0.070 & $<0.0001$ \\
\hline 10,20 & 0.130 & 0.070 & 0.0005 \\
\hline 10,30 & 0.160 & 0.067 & $<0.0001$ \\
\hline 10,60 & 0.276 & 0.070 & $<0.0001$ \\
\hline 10,90 & 0.256 & 0.073 & $<0.0001$ \\
\hline 10,120 & 0.240 & 0.070 & $<0.0001$ \\
\hline 20,30 & 0.030 & 0.070 & 0.3916 \\
\hline 20,60 & 0.146 & 0.072 & 0.0002 \\
\hline 20,90 & 0.126 & 0.075 & 0.0014 \\
\hline 20,120 & 0.111 & 0.072 & 0.0032 \\
\hline 30,60 & 0.116 & 0.070 & 0.0015 \\
\hline 30,90 & 0.096 & 0.073 & 0.0105 \\
\hline 30,120 & 0.081 & 0.070 & 0.0238 \\
\hline 60,90 & -0.021 & 0.075 & 0.5926 \\
\hline 60,120 & -0.035 & 0.072 & 0.3272 \\
\hline 90,120 & -0.015 & 0.075 & 0.6820 \\
\hline
\end{tabular}


Table 2: Pairwise comparisons between time points for light scattering intensity ratios. Shown are the mean differences in intensity ratios between time points, the critical differences required to achieve a $\mathrm{p}$-value less than 0.05 , and the $\mathrm{p}$-values as calculated by using Fisher's PLSD.

\begin{tabular}{|c|c|c|c|}
\hline Time Points (min.) & Mean Diff. & Critical Diff. & P-Value \\
\hline 0,5 & 0.029 & 0.033 & 0.0845 \\
\hline 0,10 & 0.031 & 0.032 & 0.0571 \\
\hline 0,20 & 0.054 & 0.033 & 0.0023 \\
\hline 0,30 & 0.085 & 0.032 & $<0.0001$ \\
\hline 0,60 & 0.094 & 0.033 & $<0.0001$ \\
\hline 0,90 & 0.133 & 0.033 & $<0.0001$ \\
\hline 0,120 & 0.132 & 0.033 & $<0.0001$ \\
\hline 5,10 & 0.002 & 0.032 & 0.8977 \\
\hline 5,20 & 0.024 & 0.033 & 0.1522 \\
\hline 5,30 & 0.056 & 0.032 & 0.0012 \\
\hline 5,60 & 0.065 & 0.033 & 0.0003 \\
\hline 5,90 & 0.104 & 0.033 & $<0.0001$ \\
\hline 5,120 & 0.103 & 0.033 & $<0.0001$ \\
\hline 10,20 & 0.022 & 0.032 & 0.1760 \\
\hline 10,30 & 0.054 & 0.031 & 0.0012 \\
\hline 10,60 & 0.063 & 0.032 & 0.0003 \\
\hline 10,90 & 0.102 & 0.032 & $<0.0001$ \\
\hline 10,120 & 0.101 & 0.032 & $<0.0001$ \\
\hline 20,30 & 0.031 & 0.032 & 0.5720 \\
\hline 20,60 & 0.041 & 0.033 & 0.0184 \\
\hline 20,90 & 0.080 & 0.033 & $<0.0001$ \\
\hline 20,120 & 0.078 & 0.033 & $<0.0001$ \\
\hline 30,60 & 0.009 & 0.032 & 0.5710 \\
\hline 30,90 & 0.048 & 0.032 & 0.0042 \\
\hline 30,120 & 0.047 & 0.032 & 0.0053 \\
\hline 60,90 & 0.039 & 0.033 & 0.0227 \\
\hline 60,120 & 0.038 & 0.033 & 0.0276 \\
\hline 90,120 & -0.001 & 0.033 & 0.9348 \\
\hline
\end{tabular}

